http://jmscr.igmpublication.org/home/ ISSN (e)-2347-176x ISSN (p) 2455-0450 crossref DOI: https://dx.doi.org/10.18535/jmscr/v8i4.23

\title{
A Sticky Situation
}

Authors

\section{Priya Ahire, Pramod Nichat, Kushagra Rahul*, Soumya Chatnalkar, Saad Shaikh, Geoffrey Kharmutee, Dinesh Goswami, Dhiraj Kachare}

Department of General Surgery, Grant Government Medical College, Mumbai 400008

*Corresponding Author

Dr Kushagra Rahul

Department of General Surgery, Grant Government Medical College, Mumbai 400008, India

\begin{abstract}
Introduction: Gastro- diaphragmatic injury by an ingested foreign body is of an extremely rare occurrence. It has complex anesthetic and surgical management. The authors here describe the successful surgical and anesthetic management of a case of gastric fundus perforation and diaphragmatic rent caused by ingestion of a long foreign body by the patient

Case Report: A 63 year old male patient brought by relatives to the emergency department with acute onset of dyspnea and breathlessness. Plain chest $x$ ray showed massive hydropneumothorax. Patient was resuscitated and a chest tube was inserted.

Upon ICD insertion, serous fluid was extracted. CECT of thorax and abdomen revealed a long foreign body in the stomach and perforating the fundus of the stomach, diaphragm and the lung parenchyma

This was consistent with the history of long Neem twig ingestion by the patient.

Patient was immediately operated on and the Neem stick was recovered through an iatrogenic gastrostomy and fundus wall and diaphragm were repaired. Post- operative recovery was uneventful.

Discussion: Resuscitation and close monitoring prior to and during surgery are vital. Resuscitation should be as per the ATLS protocol and immediate threats to life should be corrected before correcting the problems that do not pose immediate threats to life.

Conclusion: Gastro diaphragmatic injuries by ingested foreign bodies demand immediate life-saving measures, appropriate resuscitative care, urgent shifting of patients to tertiary care centers, prompt diagnosis and immediate surgical intervention.

Correlation of history with the presenting problem and early intervention in such cases can improve the patient outcome and minimize mortality.

Keywords: Foreign Body, Gastric Perforation, Diaphragmatic Perforation.
\end{abstract}

\section{Introduction}

Gastro- diaphragmatic injury by an ingested foreign body is of an extremely rare occurrence. It has complex anesthetic and surgical management. Urgent resuscitation and referral to a tertiary care centre is of utmost importance as such cases require a multi-disciplinary approach as cardio thoracic intervention may be required in case of mediastinal injury. Esophageal injuries must be ruled out in such cases.

Management of such cases is challenging to anaesthetists and surgeons. Intraoperatively one 
must think of the easiest and safest passage for the retrieval of the foreign body, which should be removed in toto.

Here the authors report the successful anesthetic and surgical management of a case of stomach fundus and diaphragmatic rent caused due to ingestion of a foreign body by a 63 year old male. Written informed consent was obtained from the patient for publication of this case report and any accompanying images.

\section{Case Report}

A 63 year old male patient was brought by relatives to the emergency department with acute onset of dyspnoea and breathlessness and a history of ingestion of Neem stick four days ago. On examination, he was anxious, tachycardic (124/minutes), tachypnic (Respiratory rate $38 /$ minute), normotensive (110/80mmhg) with an oxygen saturation of $78 \%$ on room air and breath sound were decreased on the left side of the chest. There was generalized tenderness over the abdomen. Bowel sounds were not audible.

Plain chest $x$ ray showed massive hydropneumothorax on the left side (Fig 1).
Patient was immediately resuscitated as per the ATLS protocol and intercostal chest tube drain (ICD) was inserted on the left side.

Upon ICD insertion, about 1.5 litres of serous fluid was drained. CECT of thorax and abdomen revealed a long foreign body in the stomach which was perforating the fundus of the stomach, diaphragm and the lung parenchyma. (Fig 2)

This was consistent with the history of long Neem twig ingestion by the patient.

CECT thorax was reviewed and mediastinal injury was ruled out.

Patient was immediately taken to the operation theatre and his abdomen was explored through a midline vertical incision. The Neem stick was palpated in the stomach and recovered through an iatrogenic gastrostomy wound.

The rent in the fundus wall was repaired with PDS sutures and diaphragmatic rent was sutured with prolene sutures. Abdominal drainage tube was placed. Thorough washes were given.

Post- operative recovery was uneventful. A thorough psychiatric analysis of the patient was done and the patient was found to be in a normal mental state without any psychiatric condition.

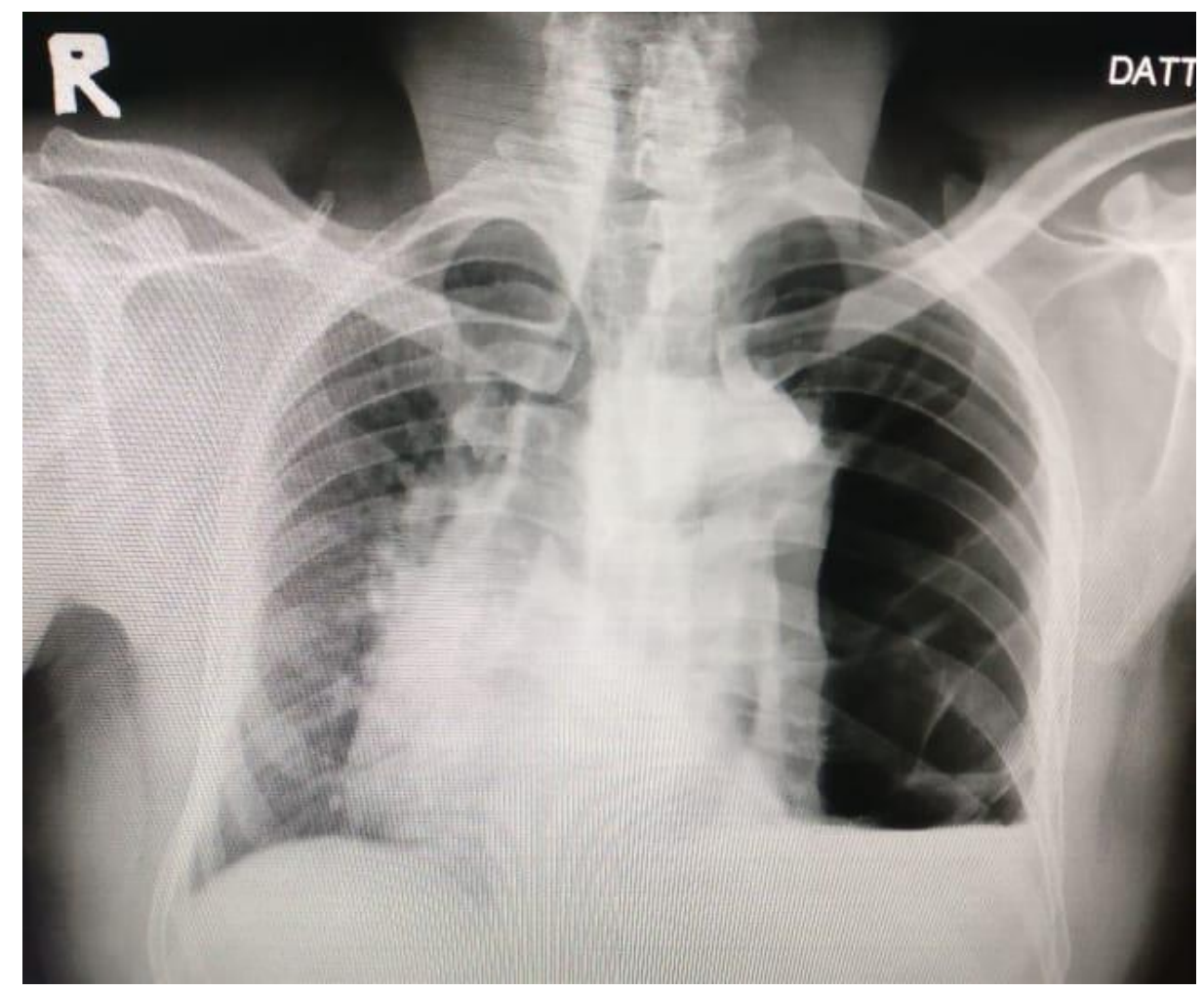

Fig 1. X ray chest showing massive hydro- pneumothorax on the left side 


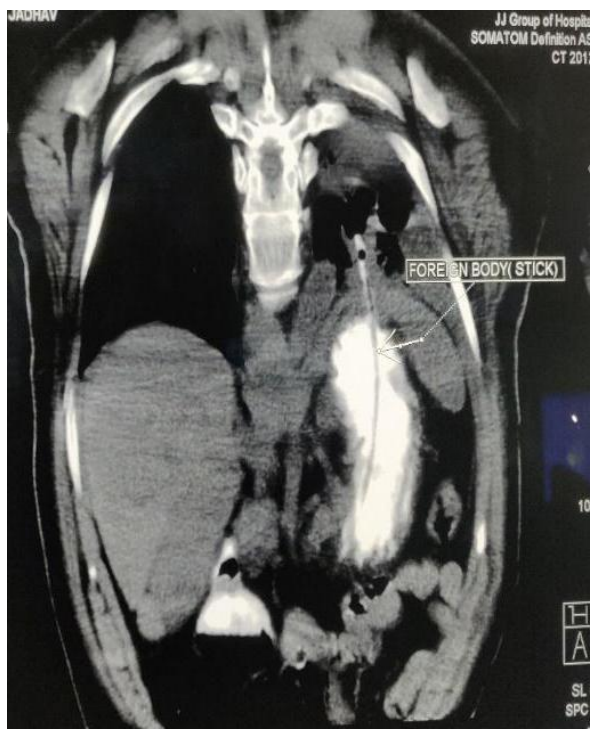

2.(a)

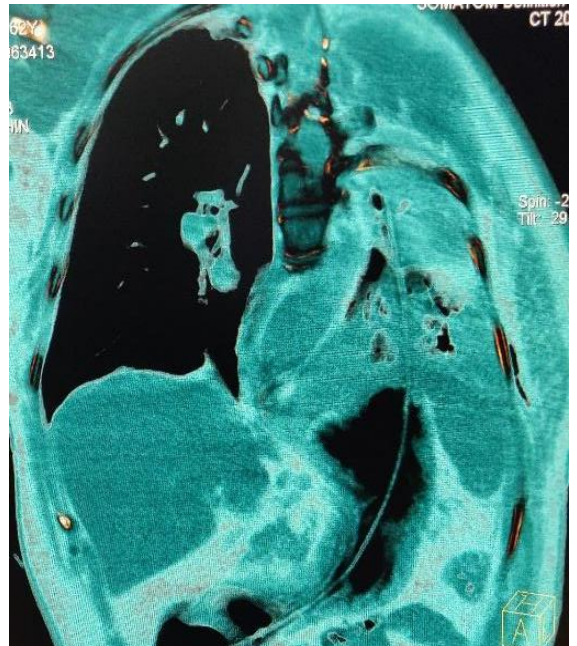

(b)

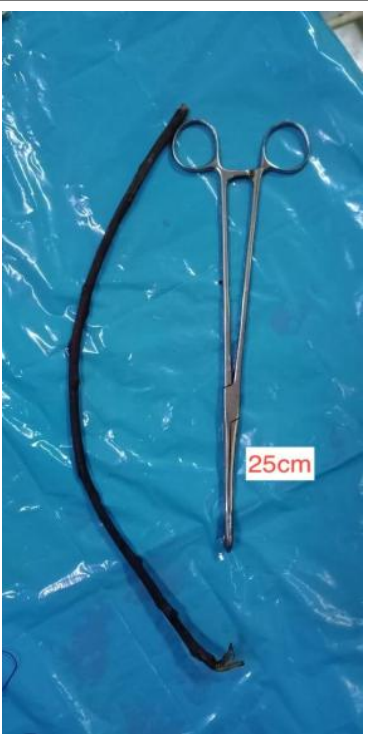

(c)

Fig. 2 a,b-CT scan showing the stick lodged into the stomach, from pylorus to fundus and perforating through the diaphragm into the lung parenchyma.

c) Stick placed next to sponge holder $(25 \mathrm{~cm})$ after retrieval

\section{Discussion}

Lack of medical education in the common population can lead to such scenarios (such as this one), where people rather than seeking medical help, go for local remedies or resort to quacks. The patient here was suffering from symptoms of acid peptic disease and used to feel heaviness in the upper abdomen. So he thought there must be some food particle lodged there which could be dislodged with a stick.

As he was manipulating his esophagus with the stick, due to peristaltic movements, he ended up ingesting a one feet long stick which later perforated its way into the lung parenchyma.

Hence as important it is to manage such conditions, it is equally important to prevent such scenarios by spreading awareness.

Early resuscitation and correlation of history of the patient with the clinical symptoms is of utmost importance. Resuscitation should be as per the ATLS protocol and immediate threats to life should be corrected before correcting the problems that do not pose immediate threats to life.

Further management of such patients must be done in a tertiary care institute where multidisciplinary teams are available.

\section{Conclusion}

Gastrothoracic perforations by ingested foreign bodies demand immediate life-saving measures, appropriate resuscitative care, urgent shifting of patient to tertiary care center, prompt diagnosis and immediate surgical intervention.

Correlation of history with the presenting problem and early intervention in such cases can improve the patient outcome and minimize mortality.

\section{References}

1. T. B. Hunter and M. S. Taljanovic, "Foreign bodies," Radiographics, vol. 23.

2. Anderson KL, Dean AJ. Foreign bodies in the gastrointestinal tract and anorectal emergencies. Emerg Med Clin North Am 2011; 29: 369-400, ix [PMID: 21515184 DOI: 10.1016/j.emc.2011.01.009].

3. Ayantunde AA, Oke T. A review of gastrointestinal foreign bodies. Int $\mathrm{J}$ Clin Pract 2006; 60: 735-739 [PMID: $16805760 \quad$ DOI: $\quad 10.1111 / \mathrm{j} .1368-$ 5031.2006.00709].

4. Ambe P, Weber SA, Schauer M, Knoefel WT. Swallowed foreign bodies in adults. Dtsch Arztebl Int 2012; 109: 869-875 
PMID:

23293675

10.3238/arztebl.2012.086].

5. Webb WA. Management of foreign bodies of the upper gastrointestinal tract: update. Gastrointestinal Endoscopic 1995; 41: 3951 [PMID: 7698623]

6. Smith MT, Wong RK. Foreign bodies. Gastrointestinal Endoscopic Clinical N Am 2007;17:361-82,vii [PMID: 17556153 DOI: 10.1016/j.giec.2007.03.002].

7. Stack LB, Munter DW. Foreign bodies in the gastrointestinal tract. Emerg Med Clin North Am 1996; 14: 493-521 [PMID: 868188].

8. Pinero Madrona A, Fernández Hernández JA, Carrasco Prats M, Riquelme Riquelme J, ParrilaParicio P. Intestinal perforation by foreign bodies. Eur J Surg 2000; 166: 307-309 [PMID: 10817327 DOI: 10.1080/110241500750009140].

9. Syrakos T, Zacharakis E, Antonitsis P, Zacharakis E, Spanos C, Georgantis G, Kiskinis D. Surgical intervention for gastrointestinal foreign bodies in adults: a case series. Med Princ Pract 2008; 17: 276-279 [PMID: 18523393 DOI: 10.1159/000129605].

10. Coulier B, Tancredi MH, Ramboux A. Spiral CT and multi-detector-row CT diagnosis of perforation of the small intestine caused by ingested foreign bodies. Eur Radiol 2004; 14: 1918-1925 [PMID: 15378256 DOI: $10.1007 / \mathrm{s} 00330-004-$ 2430-1.

11. Lyons MF, Tsuchida AM. Foreign bodies of the gastrointestinal tract. Med Clin North Am 1993; 77: 1101-1114 [PMID: 8371617].

12. ATLS 10th edition offers new insights into managing trauma patients by Sharon Henry, MD, FACS PUBLISHED JUNE 1, 2018. 